\title{
An Overview of Sleep and Circadian Rhythms in Bipolar Disorder
}

L. Bisdounis ${ }^{1,2}$, N.M. McGowan ${ }^{1} \&$ K.E.A. Saunders ${ }^{2,3}$

${ }^{1}$ Sleep and Circadian Neuroscience institute, Nuffield Department of Clinical Neurosciences, University of Oxford, United Kingdom

2 Department of Psychiatry, Warneford Hospital, University of Oxford, United Kingdom

${ }^{3}$ Oxford Health NHS Foundation Trust, Warneford Hospital, Oxford, United Kingdom

\section{Summary}

Since the conception of bipolar disorder (BD) as a psychiatric condition, sleep and circadian rhythm disruptions have been cardinal symptoms of the disorder. Current nosologies recognise this and such disruptions form part of the diagnostic criteria for both manic and depressive episodes. However, despite their historical precedence and their importance for people with $\mathrm{BD}$, sleep and circadian rhythms are often underappreciated in bipolar research. In this review we summarise key research findings about the role of sleep and circadian rhythms in BD. The areas covered include the presentation and comorbidity of sleep and circadian disorders in BD, relevant risk factors, and finally the assessment and treatment of sleep and circadian disruptions in both patients and those at-risk. Recommendations for future research focus on routine and rigorous assessment of sleep and circadian rhythms in BD, investigations into the causal ordering of sleep, circadian and mood symptoms, their use to identify and assist people at-risk of BD, and the integration of sleep and circadian interventions in treatment plans for people with BD.

Keywords: bipolar disorder, mood, sleep, circadian rhythms, insomnia 


\section{Introduction}

Bipolar Disorder (BD) is a chronic psychiatric condition characterised by alternating episodes of depression and (hypo)mania, with interim periods of subsyndromal mood symptoms, called euthymia. Typical age of onset is during adolescence or early adulthood (1), with substantial mortality and morbidity early in the illness course (2). Compared to the general population, life expectancy in BD is reduced by 8-20 years depending on the age at the time of initial diagnosis, with earlier diagnoses associated with lower life expectancy (2). BD has a global lifetime prevalence of $0.6-1 \%$ and it is associated with substantial disability, impaired psychosocial functioning, and considerable costs for the patients, their carers, and society at large (3). The clinical presentation of $\mathrm{BD}$ is highly variable. The International Classification of Diseases (ICD-11) and the Diagnostic and Statistical Manual of Mental Disorders (DSM-5) formally recognise two main subgroups within the bipolar spectrum: BD-I and BD-II $(4,5)$. BD-I features a manic and usually a depressive episode, whereas BD-II is defined by the presence of both hypomanic and depressive episodes. Across BD-I and BD-II, patients spend $50-60 \%$ of every year in an acute episode, with depressive episodes being nine times more frequent than (hypo)manic ones (6). Treatment guidelines recommend lithium as the gold standard treatment for BD with anticonvulsant, antipsychotic, or antidepressant medications also used adjunctively depending on the episodic phase of the disorder $(7,8)$. Adjunctive psychotherapy and psychoeducation is recommended for long-term maintenance and the management of subsyndromal symptoms $(7,8)$.

From the earliest conceptualisation of BD as a diagnosable condition, sleep disruption has been a hallmark of the disorder's presentation. In 1921, Emil Kraepelin wrote:

"The attacks of manic-depressive insanity are invariably accompanied by all kinds of bodily changes. By far the most striking are the disorders of sleep and general nourishment. In mania sleep is in the more severe states of excitement always considerably encroached upon; sometimes there is even almost complete sleeplessness, at most interrupted for a few hours, which may last for weeks, even months... In the states of depression in spite of great need for sleep, it is for the most part sensibly encroached upon; the patients lie for hours, sleepless in bed, ... although even in bed they find no refreshment." (9)

- Kraepelin E, Manic-Depressive Insanity and Paranoia, Edinburgh, Livingstone, 1921

Over one hundred years later, and despite undeniable advances in our understanding of BD, this notion remains relevant. Sleep and circadian disturbances are highly prevalent symptoms of BD, and salient to both patients and clinicians. Notwithstanding the uncontroversial nature of this phenomenon, such disruptions are often viewed as secondary in mental health research. In this review, we examine the evidence base for the role of sleep and circadian disturbance in $\mathrm{BD}$, extending from symptoms to prodromal signs to treatment targets. We also highlight potential key research areas for development.

\section{Sleep and Circadian Rhythms}

The human circadian system is a distributed network of autonomous cellular oscillators that produce near 24-hour rhythms, known as circadian rhythms, hierarchically controlled by the suprachiasmatic nucleus (SCN), which serves as a central pacemaker of the system. The molecular basis of cellular circadian rhythms involves a panel of key clock genes and their transcriptional/translational feedback loops. Clock genes exert an important influence over several physiologic systems and are crucial to the bottom-up operation of the circadian clock. Top-down control over the system is exerted by the SCN, the function of which is to synchronise or 'entrain' the circadian clock to the environment. The principal entraining agent of the circadian clock is environmental light, from which photic signals are transduced directly to the SCN via a class of intrinsically photoreceptive retinal ganglion cells in the retina (10). Direct interaction between the SCN and other brain regions stimulates the production of signalling molecules such as melatonin and adrenal glucocorticoids that 
may synchronize peripheral clocks. Thus, the SCN functions to orchestrate a stable phase relationship between individual clocks in the periphery and between the whole organism and the environment.

Circadian rhythm disturbance may emerge from any point of this system. For example, dysfunctional operation of clock genes and their protein products may disrupt the molecular circadian rhythms. Abnormal phase relationships within the circadian clock network may result in internal desynchrony between individual circadian rhythms. Further, inadequate environmental exposure to light or abnormal photic signalling may adversely affect circadian entrainment, and occupational challenges arising from shift-work and other work/school schedules can produce chronic mismatch between the circadian clock and the environment. In each of the above cases circadian rhythm disturbance may produce phase-advances or -delays, which in turn can severely hinder healthy sleep (11).

In terms of its definition, sleep is easily recognised on a behavioural level as a reversible state of decreased consciousness, perceptual disengagement, physical quiescence, recumbent posture, and eye closure. On a neurophysiological level, sleep is divided into two alternating stages: non rapid eye movement (NREM) and rapid eye movement (REM) sleep. NREM sleep is further subdivided into three stages of progressively increasing sleep depth (N1-3), while REM sleep is subdivided into phasic and tonic. The sleep-wake cycle is the most conspicuous process following a circadian pattern and is regulated by homeostatic and circadian principles (12). Sleep homeostasis is represented as a sleep pressure that increases during wakefulness and dissipates during sleep. If wakefulness is extended beyond the typical range, then a sleep debt is created and as a response of accumulated sleep pressure. Slow wave activity is the typical index of sleep pressure. In return, sleep homeostasis is controlled by the endogenous circadian rhythms, with core body temperature and melatonin levels being the most reliable indices of this second process.

\section{Sleep and Circadian Symptoms in BD}

The ICD-11 and DSM-5 recognise a decreased need for sleep as a diagnostic symptom of mania, and insomnia or hypersomnia as diagnostic symptoms of depression $(4,5)$. Their prevalence in acute episodes is remarkably high. Decreased need for sleep is reported 69-99\% of the time in acute (hypo)manic episodes, while up to $78 \%$ of patients in bipolar depression report hypersomnia symptoms and up to $100 \%$ report symptoms of insomnia. (13). Around 70\% of BD patients report the persistence of sleep and circadian disruption in euthymia. Increased sleep onset latency, subjective daytime dysfunction, and increased use of hypnotics have all been reported $(14,15)$. Due to the frequency and impact of these disruptions, a diagnosis of a comorbid sleep disorder is often necessary but rarely provided. It is estimated that $55 \%$ of $\mathrm{BD}$ patients warrant a comorbid diagnosis of insomnia and 30\% warrant a comorbid diagnosis of hypersomnia $(14,16)$, while it is more common for insomnia and hypersomnia symptoms to co-occur in bipolar depression, than to present independently (17). More specifically, individuals presenting with a major depressive episode who report both insomnia and hypersomnia symptoms have an up to three-fold increased risk of life-time BD (18). The co-occurrence of insomnia and hypersomnia symptoms can manifest as an inability to initiate or maintain sleep accompanied by excessive fatigue, sleepiness, anergia and increased time in bed. A caveat in our understanding of sleep disorders in $\mathrm{BD}$ is that research on the role of hypersomnia is sparse, despite evidence that it is a recurrent complaint in separate episodes of bipolar depression and studies showing that it might a useful criterion to differentiate unipolar depression and BD, especially in the case of BD-II (19).

Even less attention has been given to circadian disruptions. Across the disorder, patients consistently exhibit delayed-phase patterns and an evening chronotype, favouring a late bed- and rise-time, and report more unstable daily routines, including irregular sleep-wake cycles (20). Around one third of people with BD warrant a diagnosis of a circadian rhythms disorder, with $77 \%$ of those meeting the criteria for a delayed sleep phase disorder (21).The presence of a delayed phase has also been proposed as a characteristic feature of BD, with 
delayed sleep phase disorder being significantly more prevalent in patients with bipolar disorder compared to patients with unipolar depression and healthy controls (22).

\section{Importance of Sleep and Circadian Rhythm Disruptions in BD}

Sleep disruptions can be associated with several markers of poorer outcomes in BD. In a sample of 6,000 bipolar patients, the presence of a sleep disorder was significantly associated with an increased likelihood of suicide attempts (OR: 8.98; 95\%CI=8.24-9.80) (23). This relationship was larger than the one observed in depression, substance-use disorder, schizophrenia, post-traumatic stress disorder, and anxiety disorders (23). The presence of such disturbances has also been linked to lower quality of life in symptomatic episodes and euthymic phases, with sleep being recognised as an important contributory factor to quality of life in BD overall $(24,25)$. Sleep disturbance has been linked to self-reported decreases in productivity (24) and objective measures of reduced work performance, such as higher unemployment and being fired from work more often (26). Recent work has also highlighted that sleep disruptions might be primary causes of cognitive problems in BD. Patients with sleep disruptions perform worse in tasks of sustained attention and processing speed tasks compared to healthy controls and BD patients without such disruptions, with the latter two groups performing at an equal level (27). In addition to their adverse effects in their own rights, sleep disruptions can also exacerbate mood symptomatology in BD. Sleep related variables account for over a third of the variance in bipolar mood symptom changes over longitudinal assessments (28), and they are predictive of a shorter episode relapse even when accounting for residual mood symptoms (15). Circadian rhythm disruptions have also been described among multiple behavioural and physiologic parameters in patients with BD. Some studies also suggest that such disruptions may be an important predictor of relapse with acute circadian rhythm phaseadvances occuring before the onset of mania (29), and phase-delays before the onset of depression (30). Studies examining seasonal trends in BD episode presentation suggest that the condition displays some seasonality. Indeed, a review of health service utilisation studies for people with BD highlighted a seasonal pattern with the frequency of hospital admissions for manic and mixed episodes peaking in the spring and summer and depressive episodes peaking during the winter (31). Circadian rhythm disturbance arising from seasonal changes in the photoperiod are hypothesised to contribute to seasonal differences in hospitalisation. Knowledge of how individuals with $\mathrm{BD}$ are more sensitive to environmental circadian rhythm disturbance may have important implications for public health, especially in regions with profound fluctuation in daylength.

Most importantly, sleep and circadian rhythm function is a domain particularly salient to patients, carers and clinicians. In a survey of unipolar and bipolar depression patients, fatigue, low energy, insomnia and hypersomnia were reported among the most important outcomes of interest (32). In another study, activity, energy and sleep were the most frequently used domains for longitudinal symptom self-monitoring by BD patients (33). As such, the importance of sleep and circadian disruptions and their association with functional outcomes, render them an important target for independent assessment and treatment. Nevertheless, clinical trials in BD exceedingly focus on mood and ignore these disruptions (34), even despite their tendency to persist standard care (15).

\section{Assessment of Sleep and Circadian Rhythms in BD}

\subsection{Methods of Sleep and Circadian Assessment in BD}

As in other psychiatric disorders, the examination of sleep disruptions in BD relies on phenomenology and self-report measures. Clinical interviews and sleep diaries are the cornerstone of their assessment, while self-reported questionnaires such as the Insomnia Severity Index, the Pittsburgh Sleep Quality Inventory, the Morningness-Eveningness Questionnaire, the Sleep Hygiene Inventory and the Epsworth Sleepiness Scale are 
also common (35). As a minimum it is recommended that clinicians assess sleep quality, insomnia symptoms, chronotype, sleep hygiene, and excessive sleepiness (35). Recently, rest-activity assessments have also been used to complement such assessments. These assessments are carried out via wrist-worn accelerometer devices called actigraphs or actiwatches; a low-cost motion-sensor equipment that can capture motor activity over multiple weeks with minimal participant burden. According to a review of rest-activity research in this area, people with $\mathrm{BD}$ are less active, have lower sleep efficiency and spend more time awake than healthy controls (36). Notable limitations here are that $69 \%$ of the included studies only recruited euthymic patients and the median duration of recording was one week (36), as opposed to the general recommendation of two weeks (37). In one study looking at individuals hospitalised with $\mathrm{BD}$, it was found that those admitted with depression were less active than healthy controls and those admitted with mania, especially in the first hours of the morning (38). Conversely, inpatients with mania showed increased variance in the high frequency part of the activity spectrum, and despite the medication, their rest-activity profile generally resembled more that of patients with schizophrenia than patients with bipolar depression (38).

Neurophysiological measures of sleep are rare in BD. In a review of studies using polysomnography (i.e. a multi-parametric technique using overnight simultaneous electroencephalographic, electrooculographic and electromyographic indices, considered as the gold-standard of sleep measurement), the authors observed increased sleep latency and REM sleep density across all stages of $\mathrm{BD}$, and reduced spindle activity in N1 and N2 during depression and euthymia (39). However, two thirds of the included studies were conducted before 2,000 and there was notable variation both in the diagnosis of the patient groups as well as the conduct and scoring of the polysomnography data. In terms of circadian assessments, physiologic parameters that usually express robust circadian rhythms are found to be attenuated or expressing an abnormal circadian period in BD. For example melatonin rhythms are reduced in amplitude and delayed in phase in patients with BD (40). Evidence also points to a supersensitivity to short-wavelength light in people with BD in which they are subject to greater phase delay in melatonin synthesis; a phenotype which may contribute to non-optimally entrained circadian rhythms in the disorder (41). In people with $\mathrm{BD}$, cortisol levels upon awakening and in response to stress-invoking situations are also higher (42). Furthermore, the circadian rhythm phase of cortisol and circadian gene expression are notably different between mania and depression (29).

\subsection{Sleep and Circadian Phenotyping in BD}

$\mathrm{BD}$ is an inherently heterogeneous condition with symptom profiles varying greatly within this single diagnostic group. This issue is compounded by the poor operational definition and lack of appropriate measures for several of these symptoms. As we covered, sleep and circadian rhythms offer robust measurable outcomes for concepts related to BD. Disruptions in these areas are also ubiquitous across disorder subtype and episodic phase, while dysregulation in underlying sleep and circadian mechanisms might be shared in up to $80 \%$ of bipolar cases (43). Therefore, given their prevalence and importance, as well as the wide availability of measurement tools, sleep and circadian rhythms offer a potent domain for the clinical stratification of BD into 'treatment meaningful subgroups' (43). For example, in a recent study, polygenic risk scores for excessive daytime sleepiness and morningness were positively and negatively associated with increased odds of BD, respectively (44). However, polygenic risk scores for insomnia were associated with increased risk of BD-II but not BD-I, while polygenic risk scores for sleep duration were associated with increased odds of BD-I but not BD-II (44). Similarly, another study suggested that sleep loss might lead to worse outcomes for people with a BD-I as opposed to a BD-II diagnosis (45). Sleep and circadian rhythms appear to be intrinsic trait markers of the disorder. Cerebrospinal fluid melatonin levels are decreased across episodes in BD when compared to unipolar depression (46), in line with previous literature showing that a delayed phase might be a distinguishable marker between bipolar disorder and unipolar depression (22). 
Nevertheless, the assessment of sleep and circadian rhythms in BD is rare even among studies specifically involving these pathways (34). When present, the establishment of relevant phenotypes relies on global measures of insomnia, overlooking more granular outcomes and variables related to other prevalent disorders in BD such as hypersomnia and circadian rhythm disorders (47). Such assessments, although useful, are not conducive to the establishment of meaningful BD subgroups characterised by sleep and circadian disruption. Preliminary evidence indicates that such subgroups might be marked by a more severe clinical presentation and illness course, be more sensitive to changes in sleep and light, and respond preferentially to sleep and circadian interventions $(48,49)$

\section{Sleep and Circadian disruptions as risk factors of BD}

Transcending initial beliefs of sleep and circadian disruptions as only symptoms of BD, converging sources of evidence have demonstrated that such disturbances precede the onset of BD and the recurrence of mood episodes.

\subsection{Genetic predisposition}

Sleep and circadian gene polymorphisms have been implicated in the genetic diathesis and drug response of BD. In animals, the disruption of circadian genes has been used to model both mania (50) and depression (51). In one such model, deletion of exon 19 of the circadian gene CLOCK resulted in a dominantnegative CLOCK protein capable of binding but unable to activate transcription (50). Mice carrying this mutation exhibited increased dopaminergic activity and a behavioural profile consistent with mania, including greater risk-taking and impulsivity, hyperactivity and reduced sleep (50). Subsequent treatment with lithium or restoration of a functional CLOCK gene resulted in the dissipation of these manic-like symptoms in mutated mice (50). Over the years, several circadian candidate genes have been implicated in BD, including CLOCK, NPAS2, ARNTL1, NR1D1, PER3, RORA, RORB, CSNKe, and GSK3 $\beta$ (52). More recently, in a genome wide associations study of more than $40,000 \mathrm{BD}$ cases, BD was positively genetically correlated with measures of daytime sleepiness $(\mathrm{rg}=0.13$, $\mathrm{se}=0.03)$, insomnia $(\mathrm{rg}=0.12$, $\mathrm{se}=0.03)$, sleep duration $(\mathrm{rg}=0.12, \mathrm{se}=0.03)$, and morningness ( $\mathrm{rg}=-0.05, \mathrm{p}=0.02$ ) (53). Beyond genetic correlation, the polygenic overlap between $\mathrm{BD}$ and chronotype, sleep duration, and insomnia has been estimated at $68 \%, 73 \%$, and $86 \%$, respectively (54).

\subsection{High-risk and Prodromal Stages}

Unlike psychosis where guidelines for prodromal states have been widely established, this research is is still at its infancy in BD. Central to efforts in early identification is the concept of clinical staging (55). The notion that individuals progress through incremental and identifiable stages within a disorder continuum. In this continuum, stage 0 includes individuals at high-risk of developing the disorder but have not yet manifested any symptoms. Stage 1 represents individuals that present with non-specific subsyndromal symptoms that do not yet meet the criteria for a diagnosis. Stage 2 signifies the first episode of the disorder (here this is usually (hypo)mania given its presence is necessary to discern BD from unipolar depression), with stages 3 and 4 reflecting an established, severe and recurring disorder. In BD, stage 0 includes those with a family history of $\mathrm{BD}$, given that familial risk is the strongest predictor of later development of the disorder, with heritability estimates around $85 \%$ (56). There is a paucity of evidence to characterise individuals in stage 1 with no familial history of BD. In a prospective study including over 3,000 adolescents and young adults assessed over a period of 10 years, poor sleep quality at baseline increased the subsequent risk of receiving a diagnosis of BD by 1.75fold (57). This effect remained even after controlling for age, gender, familial history of mood disorders, as well as alcohol and cannabis use. Together with sleep, anxiety and cyclothymia (i.e. rapid oscillation in mood) have 
been proposed as phenomenological signals of the emergence of $\mathrm{BD}$ (58), but greater specificity is needed. As such, much of the focus of early identification of BD has been shwon to individuals at stage 0 .

Given this clinical staging model, children of bipolar parents comprise stage 0 and are a salient, highrisk population that can inform the scientific endeavour of identifying the emergence of BD. Cumulative research over the past two decades using bipolar offspring has indeed identified such markers of vulnerability. In large cohort studies of bipolar offspring, the presence of a sleep disorder or disruption is predictive of the development of a subsequent mood disorder. In one such study, the presence of a sleep disorder predicted a 1.63-fold increase in the risk of being diagnosed with a mood disorder in the future (59), while in another study, participants classified as poor sleepers at baseline were twice as likely to be diagnosed with BD in the future compared to participants classified as good sleepers (60). Increased energy levels and social jet lag (61), decreased need for sleep (61), increased sleep onset latency (60), irregularity of sleep patterns (62), and delayed circadian rhythms $(20,60)$ are among the sleep and circadian risk factors that emerged in prospective studies in bipolar offspring. In a systematic review that included 16 studies on high-risk and prodromal phases in BD (i.e. prospective studies on bipolar offspring, prospective studies on people with sleep disruptions that later developed $\mathrm{BD}$, or retrospective studies on people with $\mathrm{BD}$ ) sleep and circadian disruptions manifested at least a year prior to the diagnosis of BD (63). Across these studies, decreased need for sleep typically preceded manic episodes, whereas symptoms of insomnia typically preceded depressive episodes (63).

\subsection{Preceding features in episode relapse}

In people with a diagnosis of $\mathrm{BD}$, sleep and circadian disturbances have been implicated in various ways in episode relapse. Notwithstanding the lack of consensus over whether such disturbances are causal triggers or prodromal signs, they are of paramount importance in predictive models of the disorder. In a systematic review of bipolar prodromes, sleep disruptions were reported as the most robust early symptom of mania, and the sixth most frequent symptom in depression (64). Retrospective studies have also shown that life events associated with major sleep changes, such as transmeridian travelling, bereavement and childbirth, often precede the onset of mania (65). Prospective studies in this area are sparse, but there is suggestive evidence that lower and more variable total sleep time is predictive of a shorter relapse to mania and depression, respectively (66). In another prospective study, reduced sleep quality in remission was predictive of a shorter episode relapse even when controlling for residual mood symptoms (15). More robust evidence about the involvement of sleep in the multifaceted causality of BD is provided by work on total sleep deprivation. Initial reports by Thomas Wher described sleep loss as a final common patbway in the genesis of mania, owing to findings that total sleep deprivation acutely reduces depression symptoms but also leads to the emergence of symptoms of mania (67). Since then, new protocols for total sleep deprivation protocols in bipolar depression have emerged and evolved, suggesting that when the intervention is combined with lithium, the episode conversion rate is between 1.4-9\% (68).

\section{Management of Sleep and Circadian Disruptions in BD}

\subsection{Pharmacological Interventions}

Current guidelines recommend lithium as the first line of maintenance treatment, and fluoxetine and olanzapine, or quetiapine monotherapy for bipolar depression $(7,8)$. With the exception of fluoxetine, studies show that the rest of these medications might have chronotherapeutic effects $(69,70)$. Lithium response in particular is shown to be genetically determined (49), with chronotype and cellular circadian rhythms being potent markers of response. On a behavioural level, morningness at baseline predicts favourable response to lithium (71). On a molecular level, cells from lithium responsive individuals with BD exhibit a shorter circadian 
period or can be potentially shortened with lithium in vitro (71). On the other hand, cells from lithium nonresponders exhibit longer circadian periods that are less influenced by lithium administration in vitro (71). Notwithstanding, sleep and circadian rhythms are not explicitly acknowledged as direct treatment targets in BD treatment manuals, apart from the short-term use of GABA modulators for sedative purposes $(7,8)$. Owing to the involvement of circadian disruptions in $\mathrm{BD}$, melatonin and melatonin receptor agonists (e.g. agomelatine and ramelteon) have also been explored as possible treatment options. Evidence regarding their efficacy relies on a limited number of trials, with reviews suggesting that the effects of these medications on depression, mania and sleep quality are small and non-significant $(72,73)$. Recent work by our group has further highlighted that no such trial has directly examined the chronotherapeutic mechanism of action of melatonin or melatonin receptor agonists in $\mathrm{BD}$ (73). Despite presumptions of a chronobiotic mechanism of action in the justification of these trials, there is a stark absence of physiologic assessment of circadian parameters, with all trials focusing almost exclusively on assessing mood.

\subsection{Behavioural and Psychosocial Interventions}

Adjunctive psychotherapy in $\mathrm{BD}$, such as cognitive behavioural therapy and interpersonal therapy, is typically administered for long-term maintenance or to treat subsyndromal symptoms $(7,8)$. In professional treatment manuals, regularisation of sleep-wake and other daily rhythms is a recommended component of all such treatments, but never the sole treatment target $(7,8)$. Contemporary treatment manuals diverge in this respect and recognise sleep and circadian rhythms both as assessment outcomes and as independent treatment targets (74). The 2020 treatment guidelines by the Royal Australian and New Zealand College of Psychiatrists acknowledge circadian rhythms as a prominent factor in the aetiology of $\mathrm{BD}$, highlight the need for circadian assessments (i.e. chronotype, daily rhythms and sleep-wake patterns) in routine clinical practice, and recommend bright light therapy (BLT) for bipolar depression and interpersonal and social rhythm therapy (IPSRT) as a maintenance treatment (74).

Both BLT and IPSRT work via modulating circadian disturbances. Exposure to bright light in the morning or afternoon has the potential to advance the circadian phase and shift people's endogenous rhythms earlier, while IPSRT aims to stabilise people's daily routine activities. The manual also mentions the therapeutic potential of total sleep deprivation (TSD) but concludes that there is not enough evidence to recommend its general use. Other psychological and behavioural interventions that target sleep and circadian rhythms include blue-light blocking glasses, cognitive behaviour therapy for insomnia, and a combination of TSD and BLT. Reviews have reported predominantly positive findings about the efficacy of these interventions in BD (68). However, recent work from our group has challenged his notion, showing that the evidence base for the efficacy of these interventions in $\mathrm{BD}$ is limited, with previous reviews relying heavily on non-randomised and uncontrolled trials or trials looking at unipolar depression (34). The only area with enough data is regarding the effect of BLT in bipolar depression, revealing a medium-to-large reduction in depression symptoms in BLT compared to control group at post-treatment $(\mathrm{Nc}=6$; $\mathrm{g}=-0.74[95 \% \mathrm{CI}=-1.05$ to -0.42$], \mathrm{p}<0.001)$ (34). Most surprisingly, more than half of the studies included in our review (52\%) did not assess sleep or circadian rhythms in any capacity, despite this domain being the principal target of the intervention. Moreover, only $16 \%$ of the included studies recruited participants with a sleep or circadian disruption at baseline, all of which recruited BD patients with comorbid insomnia.

\subsection{Interventions in BD High-Risk}

Presently, the time between first symptom presentation and the diagnosis of BD is ten years (75), and despite the typical early onset of the disorder, individuals with late adolescent or early adult onset of BD are underrepresented in mental health services. Delays in identifying the disorder have been associated with the 
delivery of inappropriate treatment, increased hospitalisations, and elevated risk of suicide (75), emphasising the imperative for early intervention. Similar to early identification, early intervention in BD is only an emerging area, despite the wide availability of early intervention for unipolar depression and psychosis. Early interventions typically have a much lower risk-to-benefit ration, with the aim to improve functioning and delay or prevent disorder progression.

Owning to the paucity of studies looking at non-familial subsyndromal (stage 1), research on early intervention has primarily focused on people with at least one parent with a diagnosis of BD. In this population, sleep and circadian rhythms is a promising treatment target, given that it is a normative developmental process and disruptions in this domain are also common in otherwise healthy adolescents and young (55). Preliminary results show that interventions in BD high-risk youth that target the regularisation of daily rhythms lead to small but sustained improvements in sleep and mood. According to a recent systematic review, five studies have tested behavioural and psychosocial interventions in BD high-risk population with mixed findings (55). One of these studies used IPSRT (76) and two other used an intervention combing IPSRT with interpersonal family therapy $(77,78)$. IPSRT alone showed significant improvements in sleep but not in mood $(76)$, while the IPSRT combination treatments showed improvements in mood and functioning, but did not assess sleep (77, 78). Three trials also looked at pharmacological interventions in BD high-risk populations (79), with one of them testing the efficacy of lithium (80). In this trial, lithium was not more effective than placebo in altering the onset of BD in prepubescent children with probable depression and family history of BD (80).

\section{Conclusion and Future directions}

Despite the multifaceted contribution of sleep and circadian rhythms in the course of BD, and the importance of such disruptions for patients and clinicians, this domain is often underappreciated. Past work by the Committee on Chronotherapentics in Affective Disorders of the International Society for Affective Disorders, and the Chronobiology Task Force of the International Society for Bipolar Disorder has brought sleep and circadian science to the forefront and has highlighted the need of its routine integration in BD research. In this review we summarised the current state of affairs related to sleep and circadian science in BD and offered potential avenues for future research studies, namely: the routine and comprehensive assessment of sleep and circadian phenotypes, the delineation of the causal relationship between sleep, circadian rhythms and mood symptoms, the use of sleep and circadian outcomes to identify and help at-risk individuals, and the integration of sleep and circadian treatment targets in intervention research. The overarching goal is to not only to encourage research specifically on sleep and circadian rhythms, but to include chronobiological elements in all kinds of BD research. 


\section{References}

1. Bolton S, Warner J, Harriss E, Geddes J, Saunders KE. Bipolar disorder: Trimodal age-at-onset distribution. Bipolar disorders. 2021;23(4):341-56.

2. Kessing LV, Vradi E, Andersen PK. Life expectancy in bipolar disorder. Bipolar disorders. 2015;17(5):543-8.

3. McIntyre RS, Berk M, Brietzke E, Goldstein BI, López-Jaramillo C, Kessing LV, et al. Bipolar disorders. The Lancet. 2020;396(10265):1841-56.

4. American Psychiatric Association. Diagnostic and statistical manual of mental disorders (DSM-5®): American Psychiatric Pub; 2013.

5. World Health Organization. ICD-11: International classification of diseases (11th revision) https://icd.who.int/2019 [

6. McKnight RF, Bilderbeck AC, Miklowitz DJ, Hinds C, Goodwin GM, Geddes JR. Longitudinal mood monitoring in bipolar disorder: course of illness as revealed through a short messaging service. Journal of Affective Disorders. 2017;223:139-45.

7. Goodwin G, Haddad P, Ferrier I, Aronson J, Barnes T, Cipriani A, et al. Evidence-based guidelines for treating bipolar disorder: revised third edition recommendations from the British Association for Psychopharmacology. Journal of Psychopharmacology. 2016;30(6):495-553.

8. Yatham LN, Kennedy SH, Parikh SV, Schaffer A, Bond DJ, Frey BN, et al. Canadian Network for Mood and Anxiety Treatments (CANMAT) and International Society for Bipolar Disorders (ISBD) 2018 guidelines for the management of patients with bipolar disorder. Bipolar disorders. 2018;20(2):97-170.

9. Kraepelin E, Robertson GM. Manic-depressive insanity and paranoia. Edinburgh: E. \& S. Livingstone; 1921.

10. Foster RG, Kreitzman L. The rhythms of life: what your body clock means to you! Experimental physiology. 2014;99(4):599-606.

11. Roenneberg T, Merrow M. The circadian clock and human health. Current biology. 2016;26(10):R432R43.

12. Borbély AA, Daan S, Wirz-Justice A, Deboer T. The two-process model of sleep regulation: a reappraisal. Journal of sleep research. 2016;25(2):131-43.

13. Harvey AG. Sleep and circadian rhythms in bipolar disorder: seeking synchrony, harmony, and regulation. American journal of psychiatry. 2008;165(7):820-9.

14. Harvey AG, Schmidt DA, Scarnà A, Semler CN, Goodwin GM. Sleep-related functioning in euthymic patients with bipolar disorder, patients with insomnia, and subjects without sleep problems. American Journal of Psychiatry. 2005;162(1):50-7.

15. Cretu JB, Culver JL, Goffin KC, Shah S, Ketter TA. Sleep, residual mood symptoms, and time to relapse in recovered patients with bipolar disorder. Journal of affective disorders. 2016;190:162-6.

16. Grigolon RB, Trevizol AP, Cerqueira RO, Lee Y, Mansur RB, McIntyre RS, et al. Hypersomnia and bipolar disorder: A systematic review and meta-analysis of proportion. Journal of affective disorders. 2019;246:659-66.

17. Soehner AM, Kaplan KA, Harvey AG. Prevalence and clinical correlates of co-occurring insomnia and hypersomnia symptoms in depression. Journal of affective disorders. 2014;167:93-7.

18. Geoffroy PA, Hoertel N, Etain B, Bellivier F, Delorme R, Limosin F, et al. Insomnia and hypersomnia in major depressive episode: prevalence, sociodemographic characteristics and psychiatric comorbidity in a population-based study. Journal of affective disorders. 2018;226:132-41.

19. Kaplan KA, Harvey AG. Hypersomnia across mood disorders: a review and synthesis. Sleep medicine reviews. 2009;13(4):275-85.

20. Melo MC, Abreu RL, Neto VBL, de Bruin PF, de Bruin VM. Chronotype and circadian rhythm in bipolar disorder: a systematic review. Sleep medicine reviews. 2017;34:46-58.

21. Takaesu Y, Inoue Y, Murakoshi A, Komada Y, Otsuka A, Futenma K, et al. Prevalence of circadian rhythm sleep-wake disorders and associated factors in euthymic patients with bipolar disorder. PloS one. 2016;11(7):e0159578.

22. Takaesu Y. Circadian rhythm in bipolar disorder: a review of the literature. Psychiatry and clinical neurosciences. 2018;72(9):673-82. 
23. Bishop TM, Walsh PG, Ashrafioun L, Lavigne JE, Pigeon WR. Sleep, suicide behaviors, and the protective role of sleep medicine. Sleep Medicine. 2020;66:264-70.

24. Gruber J, Harvey AG, Wang PW, Brooks III JO, Thase ME, Sachs GS, et al. Sleep functioning in relation to mood, function, and quality of life at entry to the Systematic Treatment Enhancement Program for Bipolar Disorder (STEP-BD). Journal of affective disorders. 2009;114(1-3):41-9.

25. De la Fuente-Tomás L, Sierra P, Sanchez-Autet M, García-Blanco A, Safont G, Arranz B, et al. Sleep disturbances, functioning, and quality of life in euthymic patients with bipolar disorder. Psychiatry Research. 2018;269:501-7.

26. Bessonova L, Ogden K, Doane MJ, O'Sullivan AK, Tohen M. The economic burden of bipolar disorder in the United States: a systematic literature review. Clinicoeconomics and Outcomes Research: CEOR. 2020;12:481.

27. Bradley A, Anderson K, Gallagher P, McAllister-Williams RH. The association between sleep and cognitive abnormalities in bipolar disorder. Psychological Medicine. 2020;50(1):125-32.

28. Soehner AM, Bertocci MA, Levenson JC, Goldstein TR, Rooks B, Merranko J, et al. Longitudinal associations between sleep patterns and psychiatric symptom severity in high-risk and community comparison youth. Journal of the American Academy of Child \& Adolescent Psychiatry. 2019;58(6):608-17.

29. Moon J-H, Cho C-H, Son GH, Geum D, Chung S, Kim H, et al. Advanced circadian phase in mania and delayed circadian phase in mixed mania and depression returned to normal after treatment of bipolar disorder. EBioMedicine. 2016;11:285-95.

30. Steinan MK, Morken G, Lagerberg TV, Melle I, Andreassen OA, Vaaler AE, et al. Delayed sleep phase: An important circadian subtype of sleep disturbance in bipolar disorders. Journal of affective disorders. 2016;191:156-63.

31. Geoffroy PA, Bellivier F, Scott J, Etain B. Seasonality and bipolar disorder: a systematic review, from admission rates to seasonality of symptoms. Journal of Affective Disorders. 2014;168:210-23.

32. Chevance A, Ravaud P, Tomlinson A, Le Berre C, Teufer B, Touboul S, et al. Identifying outcomes for depression that matter to patients, informal caregivers, and health-care professionals: qualitative content analysis of a large international online survey. The Lancet Psychiatry. 2020;7(8):692-702.

33. Gordon-Smith K, Saunders KE, Savage J, Craddock N, Jones I, Jones L. Have I argued with my family this week?": What questions do those with lived experience choose to monitor their bipolar disorder? Journal of Affective Disorders. 2021;281:918-25.

34. Bisdounis L, Saunders KE, Farley HJ, Lee CK, McGowan NM, Espie CA, et al. Psychological and behavioural interventions in bipolar disorder that target sleep and circadian rhythms: a systematic review of randomised controlled trials. Neuroscience \& Biobehavioral Reviews. 2022;132:378-90.

35. Morton E, Murray G. Assessment and treatment of sleep problems in bipolar disorder-A guide for psychologists and clinically focused review. Clinical Psychology \& Psychotherapy. 2020;27(3):364-77.

36. De Crescenzo F, Economou A, Sharpley AL, Gormez A, Quested DJ. Actigraphic features of bipolar disorder: a systematic review and meta-analysis. Sleep medicine reviews. 2017;33:58-69.

37. Smith MT, McCrae CS, Cheung J, Martin JL, Harrod CG, Heald JL, et al. Use of actigraphy for the evaluation of sleep disorders and circadian rhythm sleep-wake disorders: an American Academy of Sleep Medicine clinical practice guideline. Journal of Clinical Sleep Medicine. 2018;14(7):1231-7.

38. Krane-Gartiser K, Henriksen TEG, Morken G, Vaaler A, Fasmer OB. Actigraphic assessment of motor activity in acutely admitted inpatients with bipolar disorder. PloS one. 2014;9(2):e89574.

39. Zangani C, Casetta C, Saunders AS, Donati F, Maggioni E, D'Agostino A. Sleep abnormalities across different clinical stages of Bipolar Disorder: A review of EEG studies. Neuroscience \& Biobehavioral Reviews. 2020;118:247-57.

40. Bradley A, Webb-Mitchell R, Hazu A, Slater N, Middleton B, Gallagher P, et al. Sleep and circadian rhythm disturbance in bipolar disorder. Psychological medicine. 2017;47(9):1678-89.

41. Ritter P, Soltmann B, Sauer C, Yakac A, Boekstaegers L, Reichard M, et al. Supersensitivity of Patients With Bipolar I Disorder to Light-Induced Phase Delay by Narrow Bandwidth Blue Light. Biological Psychiatry Global Open Science. 2022;2(1):28-35. 
42. Girshkin L, O'Reilly N, Quidé Y, Teroganova N, Rowland JE, Schofield PR, et al. Diurnal cortisol variation and cortisol response to an MRI stressor in schizophrenia and bipolar disorder. Psychoneuroendocrinology. 2016;67:61-9.

43. Carpenter JS, Crouse JJ, Scott EM, Naismith SL, Wilson C, Scott J, et al. Circadian depression: a mood disorder phenotype. Neuroscience \& Biobehavioral Reviews. 2021;126:79-101.

44. Lewis KJ, Richards A, Karlsson R, Leonenko G, Jones SE, Jones HJ, et al. Comparison of genetic liability for sleep traits among individuals with bipolar disorder I or II and control participants. JAMA psychiatry. 2020;77(3):303-10.

45. Lewis KS, Gordon-Smith K, Forty L, Di Florio A, Craddock N, Jones L, et al. Sleep loss as a trigger of mood episodes in bipolar disorder: individual differences based on diagnostic subtype and gender. The British Journal of Psychiatry. 2017;211(3):169-74.

46. Bumb J, Enning F, Mueller J, van der List T, Rohleder C, Findeisen P, et al. Differential melatonin alterations in cerebrospinal fluid and serum of patients with major depressive disorder and bipolar disorder. Comprehensive psychiatry. 2016;68:34-9.

47. Kaplan KA, Williams R. Hypersomnia: an overlooked, but not overestimated, sleep disturbance in bipolar disorder. Evidence-Based Mental Health. 2017;20(2):59-.

48. Takaesu Y, Inoue Y, Ono K, Murakoshi A, Futenma K, Komada Y, et al. Circadian rhythm sleep-wake disorders predict shorter time to relapse of mood episodes in euthymic patients with bipolar disorder: a prospective 48-week study. The Journal of clinical psychiatry. 2017;78(1):2651.

49. McCarthy MJ, Gottlieb JF, Gonzalez R, McClung CA, Alloy LB, Cain S, et al. Neurobiological and behavioral mechanisms of circadian rhythm disruption in bipolar disorder: A critical multi-disciplinary literature review and agenda for future research from the ISBD task force on chronobiology. Bipolar disorders. 2021.

50. Roybal K, Theobold D, Graham A, DiNieri JA, Russo SJ, Krishnan V, et al. Mania-like behavior induced by disruption of CLOCK. Proceedings of the National Academy of Sciences. 2007;104(15):6406-11.

51. Landgraf D, Long JE, Proulx CD, Barandas R, Malinow R, Welsh DK. Genetic disruption of circadian rhythms in the suprachiasmatic nucleus causes helplessness, behavioral despair, and anxiety-like behavior in mice. Biological psychiatry. 2016;80(11):827-35.

52. Geoffroy PA. Clock genes and light signaling alterations in bipolar disorder: when the biological clock is off. Biological psychiatry. 2018;84(11):775-7.

53. Mullins N, Forstner AJ, O'Connell KS, Coombes B, Coleman JR, Qiao Z, et al. Genome-wide association study of more than 40,000 bipolar disorder cases provides new insights into the underlying biology. Nature genetics. 2021;53(6):817-29.

54. O'Connell KS, Frei O, Bahrami S, Smeland OB, Bettella F, Cheng W, et al. Characterizing the genetic overlap between psychiatric disorders and sleep-related phenotypes. Biological Psychiatry. 2021;90(9):621-31.

55. Vallarino M, Henry C, Etain B, Gehue LJ, Macneil C, Scott EM, et al. An evidence map of psychosocial interventions for the earliest stages of bipolar disorder. The Lancet Psychiatry. 2015;2(6):548-63.

56. Bienvenu OJ, Davydow DS, Kendler K. Psychiatric 'diseases' versus behavioral disorders and degree of genetic influence. Psychological medicine. 2011;41(1):33-40.

57. Ritter PS, Höfler M, Wittchen H-U, Lieb R, Bauer M, Pfennig A, et al. Disturbed sleep as risk factor for the subsequent onset of bipolar disorder-data from a 10-year prospective-longitudinal study among adolescents and young adults. Journal of psychiatric research. 2015;68:76-82.

58. Kapczinski F, Magalhães P, Balanzá-Martinez V, Dias V, Frangou S, Gama C, et al. Staging systems in bipolar disorder: an I nternational S ociety for B ipolar D isorders $\mathrm{T}$ ask $\mathrm{F}$ orce $\mathrm{R}$ eport. Acta psychiatrica scandinavica. 2014;130(5):354-63.

59. Duffy A, Goodday S, Keown-Stoneman C, Grof P. The emergent course of bipolar disorder: observations over two decades from the Canadian high-risk offspring cohort. American Journal of Psychiatry. 2019;176(9):720-9.

60. Levenson JC, Soehner A, Rooks B, Goldstein TR, Diler R, Merranko J, et al. Longitudinal sleep phenotypes among offspring of bipolar parents and community controls. Journal of affective disorders. 2017;215:30-6.

61. Sebela A, Kolenic M, Farkova E, Novak T, Goetz M. Decreased need for sleep as an endophenotype of bipolar disorder: an actigraphy study. Chronobiology international. 2019;36(9):1227-39. 
62. Singh MK, DelBello MP, Strakowski SM. Temperament in child offspring of parents with bipolar disorder. Journal of Child and Adolescent Psychopharmacology. 2008;18(6):589-93.

63. Pancheri C, Verdolini N, Pacchiarotti I, Samalin L, Delle Chiaie R, Biondi M, et al. A systematic review on sleep alterations anticipating the onset of bipolar disorder. European Psychiatry. 2019;58:45-53.

64. Jackson A, Cavanagh J, Scott J. A systematic review of manic and depressive prodromes. Journal of affective disorders. 2003;74(3):209-17.

65. Plante DT, Winkelman JW. Sleep disturbance in bipolar disorder: therapeutic implications. American Journal of Psychiatry. 2008;165(7):830-43.

66. Gruber J, Miklowitz DJ, Harvey AG, Frank E, Kupfer D, Thase ME, et al. Sleep matters: sleep functioning and course of illness in bipolar disorder. Journal of affective disorders. 2011;134(1-3):416-20.

67. Wehr TA, Sack DA, Rosenthal NE. Sleep reduction as a final common pathway in the genesis of mania. The American journal of psychiatry. 1987.

68. Gottlieb JF, Benedetti F, Geoffroy PA, Henriksen TE, Lam RW, Murray G, et al. The chronotherapeutic treatment of bipolar disorders: A systematic review and practice recommendations from the ISBD task force on chronotherapy and chronobiology. Bipolar disorders. 2019;21(8):741-73.

69. Rock PL, Goodwin GM, Wulff K, McTavish SF, Harmer CJ. Effects of short-term quetiapine treatment on emotional processing, sleep and circadian rhythms. Journal of Psychopharmacology. 2016;30(3):273-82.

70. Sharpley AL, Attenburrow ME, Hafizi S, Cowen PJ. Olanzapine increases slow wave sleep and sleep continuity in SSRI-resistant depressed patients. The Journal of clinical psychiatry. 2005;66(4):13139.

71. McCarthy MJ. Missing a beat: assessment of circadian rhythm abnormalities in bipolar disorder in the genomic era. Psychiatric genetics. 2019;29(2):29-36.

72. Kishi T, Nomura I, Sakuma K, Kitajima T, Mishima K, Iwata N. Melatonin receptor agonistsramelteon and melatonin-for bipolar disorder: a systematic review and meta-analysis of double-blind, randomized, placebo-controlled trials. Neuropsychiatric disease and treatment. 2019;15:1479.

73. McGowan NM KD, de Andres Crespo M, Bisdounis L, Kyle SD, and Saunders KEA,. Hypnotic and melatonin/melatonin-receptor agonist treatment in bipolar disorder: a systematic review and meta-analysis. CNS Drugs. (under review).

74. Malhi GS, Bell E, Bassett D, Boyce P, Bryant R, Hazell P, et al. The 2020 Royal Australian and New Zealand College of Psychiatrists clinical practice guidelines for mood disorders. Australian \& New Zealand Journal of Psychiatry. 2021;55(1):7-117.

75. Keck Jr PE, Kessler RC, Ross R. Clinical and economic effects of unrecognized or inadequately treated bipolar disorder. Journal of Psychiatric Practice®. 2008;14:31-8.

76. Goldstein TR, Fersch-Podrat R, Axelson DA, Gilbert A, Hlastala SA, Birmaher B, et al. Early intervention for adolescents at high risk for the development of bipolar disorder: pilot study of Interpersonal and Social Rhythm Therapy (IPSRT). Psychotherapy. 2014;51(1):180.

77. Miklowitz DJ, Chang KD, Taylor DO, George EL, Singh MK, Schneck CD, et al. Early psychosocial intervention for youth at risk for bipolar I or II disorder: A one-year treatment development trial. Bipolar Disorders. 2011;13(1):67-75.

78. Miklowitz DJ, Schneck CD, Singh MK, Taylor DO, George EL, Cosgrove VE, et al. Early intervention for symptomatic youth at risk for bipolar disorder: a randomized trial of family-focused therapy. Journal of the American Academy of Child \& Adolescent Psychiatry. 2013;52(2):121-31.

79. Saraf G, Moazen-Zadeh E, Pinto JV, Ziafat K, Torres IJ, Kesavan M, et al. Early intervention for people at high risk of developing bipolar disorder: a systematic review of clinical trials. The Lancet Psychiatry. 2021;8(1):64-75.

80. Geller B, Cooper TB, Zimerman B, Frazier J, Williams M, Heath J, et al. Lithium for prepubertal depressed children with family history predictors of future bipolarity: a double-blind, placebo-controlled study. Journal of Affective Disorders. 1998;51(2):165-75. 